\title{
The Counteracting Effects of Exercise on High-Fat Diet-Induced Memory Impairment: A Systematic Review
}

\author{
Paul D. Loprinzi ${ }^{1}$ (1), Pamela Ponce ${ }^{1}$, Liye Zou ${ }^{2}$ (I) and Hong $\mathrm{Li}^{2,3,4, *}$ \\ 1 Exercise \& Memory Laboratory, Department of Health, Exercise Science and Recreation Management, The \\ University of Mississippi, Oxford, MS 38677, USA; pdloprin@olemiss.edu (P.D.L.); pponce@uthsc.edu (P.P.) \\ 2 Shenzhen Key Laboratory of Affective and Social Cognitive Science, College of Psychology and Sociology, \\ Shenzhen University, Shenzhen 518060, China; liyezou123@gmail.com \\ 3 Research Centre of Brain Function and Psychological Science, Shenzhen University, Shenzhen 518060, China \\ 4 Shenzhen Institute of Neuroscience, Shenzhen University, Shenzhen 518060, China \\ * Correspondence: lihongszu@szu.edu.cn
}

Received: 15 May 2019; Accepted: 18 June 2019; Published: 20 June 2019

check for updates

\begin{abstract}
The objective of the present review was to evaluate whether exercise can counteract a potential high-fat diet-induced memory impairment effect. The evaluated databases included: Google Scholar, Sports Discus, Embase/PubMed, Web of Science, and PsychInfo. Studies were included if: (1) an experimental/intervention study was conducted, (2) the experiment/intervention included both a high-fat diet and exercise group, and evaluated whether exercise could counteract the negative effects of a high-fat diet on memory, and (3) evaluated memory function (any type) as the outcome measure. In total, 17 articles met the inclusionary criteria. All 17 studies (conducted in rodents) demonstrated that the high-fat diet protocol impaired memory function and all 17 studies demonstrated a counteracting effect with chronic exercise engagement. Mechanisms of these robust effects are discussed herein.
\end{abstract}

Keywords: cytokines; hippocampal neurogenesis; inflammation; insulin resistance; obesity

\section{Introduction}

Unlike traditional advice that promotes a low-fat diet [1], recently, high-fat diets (HFDs) are gaining popularity among athletes [2] and the general population [3]. However, HFDs have been shown to impair episodic memory function [4,5]. In humans, episodic memory function refers to the retrospective recall of information from a spatial-temporal context [6]. That is, episodic memory, a contextual-based memory, involves what, where, and when aspects of a memory [7]. In rodents, however, episodic memory is primarily evaluated from a spatial memory task, such as the Morris water maze task or a T-maze task.

As discussed elsewhere [8], a cellular correlate of episodic memory is long-term potentiation (LTP), a form of activity-dependent plasticity that results in enhancement of synaptic transmission [9]. The complementary process of LTP is long-term depression (LTD), in which the efficacy of synaptic transmission is reduced [10]. It is thought that LTP and LTD play an important role in memory as LTP- and LTD-like changes in synaptic strength occur as memories are formed at various sets of brain synapses [11-13]. The adverse episodic memory effects from an HFD may, in part, occur through alterations in processes that influence synaptic transmission and production of plasticity-related proteins [14-16]. For example, research demonstrates that a chronic HFD impairs hippocampal dendritic spine density [17], induces astrocyte alterations [18], reduces expression of the NR2B 
subunit of NMDA receptors [19], decreases CREB expression [20], and reduces hippocampal BDNF production [21].

Of central interest to this review is whether exercise can counteract HFD-induced memory impairment. Such an effect is plausible for several reasons. We speculate that this counteracting effect may occur from exercise activating some of the neurophysiological pathways that are involved in episodic memory function (e.g., BDNF). Further, we speculate that exercise may counteract HFD-induced memory impairment by, not only activating these pathways, but by also inhibiting the downregulation of these pathways induced by HFD. First, chronic exercise has been shown to enhance episodic memory function [22] and LTP [23]. Chronic exercise may subserve episodic memory function via inducing neurogenesis $[23,24]$ and altering LTP-related receptor (e.g., NMDA) structure and function $[25,26]$.

Couched within the above, HFD may impair episodic memory and exercise has been shown to enhance episodic memory function. Further, exercise has been shown to regulate processes (e.g., LTP) that are impaired with HFD. Thus, the specific research question of this systematic review was to evaluate the extant literature to determine whether exercise can counteract a potential HFD-induced memory impairment effect.

\section{Methods}

\subsection{Computerized Searches}

The evaluated databases included: Google Scholar, Sports Discus, Embase/PubMed, Web of Science, and PsychInfo [27]. Articles were retrieved from inception to 22 April 2019. The search terms, including their combinations, were: exercise, physical activity, diet, high-fat, memory, cognition, and cognitive function.

\subsection{Study Selection}

The computerized searches were performed separately by two authors and comparisons were made to render the number of eligible studies. Consensus was reached from these separate reviews. After conducting the searches, the article titles and abstracts were evaluated to identify applicable articles. Articles meeting the inclusionary criteria were retrieved and evaluated at the full text level.

\subsection{Inclusionary Criteria}

Studies were included if: (1) an experimental/intervention study was conducted, (2) the experiment/intervention included both an HFD and exercise group, and evaluated whether exercise could counteract the negative effects of an HFD on memory, and (3) evaluated memory function (any type) as the outcome measure.

\subsection{Data Extraction of Included Studies}

Detailed information from each of the included studies were extracted, including the following information: author, subject characteristics, exercise protocol, diet protocol, temporal assessment of the exercise and diet protocols, memory assessment, whether the diet protocol impaired memory, whether exercise counteracted the diet-induced memory impairment, and evaluated mechanisms of this attenuation effect.

\section{Results}

\subsection{Retrieved Articles}

The computerized searches identified 448 articles. Among the 448 articles, 430 were excluded and 18 full text articles were reviewed. Among these 18 articles, 1 was ineligible as it did not meet our study criteria. Thus, in total, 17 articles met the inclusionary criteria and were evaluated herein. 


\subsection{Article Synthesis}

Details on the study characteristics are displayed in Table 1 (extraction table). As shown in Table 1, all studies employed an exercise and diet paradigm in an animal model. The daily exercise protocol ranged from 6 weeks to 23 weeks. Similarly, the diet protocol ranged from 6 weeks to 23 weeks, with the majority of studies implementing an ad libitum diet consisting of $60 \%$ fat, $20 \%$ carbohydrate, and $20 \%$ protein. Across the 17 studies, there was variability on the temporal assessment of the exercise and diet protocols, consisting of either having both protocols occur concurrently or exercise occurring after the high-fat diet (treatment paradigm). Among the 17 studies, 10 implemented a concurrent model, whereas 7 implemented a treatment paradigm. Regarding the memory outcome, the majority of studies utilized a Morris water maze task, with others employing an avoidance task (e.g., passive or step-down) or a maze task (e.g., y-maze task, radial maze task, evaluated plus maze task).

Regarding the effects of HFD on memory, all 17 studies demonstrated that the HFD protocol impaired memory function. Notably, in one study, this impairment effect only occurred among a subgroup of animals (adolescent mice) [28]. Similarly, all 17 studies demonstrated that chronic exercise engagement counteracted HFD-induced memory impairment. Notably, however, one study showed that this attenuation effect only occurred if the chronic exercise protocol occurred during the majority of the HFD period [29]. 
Table 1. Extraction table of the evaluated studies.

\begin{tabular}{|c|c|c|c|c|c|c|c|c|}
\hline Study & Subjects & Exercise Protocol & Diet Protocol & $\begin{array}{l}\text { Temporal } \\
\text { Assessment of } \\
\text { Exercise and } \\
\text { Diet }\end{array}$ & $\begin{array}{c}\text { Memory } \\
\text { Assessment }\end{array}$ & $\begin{array}{l}\text { Did High-Fat } \\
\text { Diet Impair } \\
\text { Memory? }\end{array}$ & $\begin{array}{c}\text { Did Exercise } \\
\text { Counteract } \\
\text { Diet-Induced } \\
\text { Memory } \\
\text { Impairment? }\end{array}$ & Mechanisms \\
\hline $\begin{array}{l}\text { Molteni et al. } \\
\text { (2004) [4] }\end{array}$ & $\begin{array}{l}\text { Fisher } 344 \text { rats, } 2 \\
\text { months old }\end{array}$ & $\begin{array}{c}\text { Free access to } \\
\text { running wheel for } 2 \\
\text { months. }\end{array}$ & $\begin{array}{l}2 \text { months on high in } \\
\text { saturated and } \\
\text { monounsaturated fat } \\
\text { (primarily from lard } \\
\text { plus a small amount } \\
\text { of corn oil, approx. } \\
39 \% \text { energy) }\end{array}$ & Concurrent & $\begin{array}{l}\text { Morris water } \\
\text { maze }\end{array}$ & Yes & Yes & $\begin{array}{l}\text { Exercise reversed the } \\
\text { decrease in BDNF and } \\
\text { its downstream effector, } \\
\text { synapsin I (involved in } \\
\text { BDNF release). Exercise } \\
\text { also increase CREB } \\
\text { transcription. }\end{array}$ \\
\hline $\begin{array}{l}\text { Maesako et al. } \\
\text { (2012) [5] }\end{array}$ & $\begin{array}{c}\text { APP transgenic } \\
\text { mice } \\
\text { overexpressing the } \\
\text { familial AD-linked } \\
\text { mutation }\end{array}$ & $\begin{array}{l}\text { Enriched } \\
\text { environment with } \\
\text { access to running } \\
\text { wheel; this occurred } \\
\text { from weeks } 10-20 \\
\text { (i.e., } 10 \text { weeks into } \\
\text { the high-fat diet). }\end{array}$ & $\begin{array}{l}20 \text { weeks of high-fat } \\
\text { diet, involving } 60 \% \\
\text { fat, } 20 \% \text { CHO, and } \\
20 \% \text { protein }\end{array}$ & Concurrent & $\begin{array}{l}\text { Morris water } \\
\text { maze }\end{array}$ & Yes & Yes & $\begin{array}{c}\text { Enriched environment } \\
\text { attenuated diet-induced } \\
\mathrm{A} \beta \text { deposition. }\end{array}$ \\
\hline $\begin{array}{l}\text { Maesako et al. } \\
\text { (2012) [30] }\end{array}$ & $\begin{array}{c}\text { APP transgenic } \\
\text { mice } \\
\text { overexpressing the } \\
\text { familial AD-linked } \\
\text { mutation }\end{array}$ & $\begin{array}{l}\text { Voluntary access to } \\
\text { running wheel. }\end{array}$ & $\begin{array}{c}20 \text { weeks of high-fat } \\
\text { diet, involving } 60 \% \\
\text { fat, } 20 \% \text { CHO, and } \\
20 \% \text { protein }\end{array}$ & Concurrent & $\begin{array}{l}\text { Morris water } \\
\text { maze }\end{array}$ & Yes & Yes & $\begin{array}{l}\text { Exercise attenuated } \\
\text { diet-induced } A \beta \\
\text { deposition and } \\
\text { strengthened the } \\
\text { activity of neprilysin, } \\
\text { the } A \beta \text {-degrading } \\
\text { enzyme. }\end{array}$ \\
\hline $\begin{array}{l}\text { Maesako et al. } \\
\text { (2013) [29] }\end{array}$ & $\begin{array}{c}\text { APP transgenic } \\
\text { mice } \\
\text { overexpressing the } \\
\text { familial AD-linked } \\
\text { mutation }\end{array}$ & $\begin{array}{l}\text { Voluntary access to } \\
\text { running wheel. }\end{array}$ & $\begin{array}{l}20 \text { weeks of high-fat } \\
\text { diet, involving } 60 \% \\
\text { fat, } 20 \% \text { CHO, and } \\
20 \% \text { protein }\end{array}$ & Concurrent & $\begin{array}{l}\text { Morris water } \\
\text { maze }\end{array}$ & Yes & $\begin{array}{l}\text { Yes, but only if } \\
\text { the exercise } \\
\text { occurred } \\
\text { throughout the } \\
\text { majority of the } \\
\text { diet protocol }\end{array}$ & \\
\hline $\begin{array}{l}\text { Woo et al. (2013) } \\
\text { [31] }\end{array}$ & $\begin{array}{l}\text { 4-week-old } \\
\text { Sprague-Dawley } \\
\text { rats }\end{array}$ & $\begin{array}{l}\text { Treadmill exercise } \\
\text { for the first } 8 \text { weeks, } \\
\text { involving a } \\
\text { progressive exercise } \\
\text { program, ranging } \\
\text { from } 40 \text { to } 60 \\
\text { min/day of exercise. }\end{array}$ & $\begin{array}{l}\text { 13-weeks of high-fat } \\
\text { diet, involving } 45 \% \\
\text { fat }\end{array}$ & Concurrent & $\begin{array}{l}\text { Morris water } \\
\text { maze }\end{array}$ & Yes & Yes & $\begin{array}{l}\text { Upregulation of BDNF } \\
\text { and MAPK. }\end{array}$ \\
\hline
\end{tabular}


Table 1. Cont

\begin{tabular}{|c|c|c|c|c|c|c|c|c|}
\hline Study & Subjects & Exercise Protocol & Diet Protocol & $\begin{array}{c}\text { Temporal } \\
\text { Assessment of } \\
\text { Exercise and } \\
\text { Diet }\end{array}$ & $\begin{array}{c}\text { Memory } \\
\text { Assessment }\end{array}$ & $\begin{array}{l}\text { Did High-Fat } \\
\text { Diet Impair } \\
\text { Memory? }\end{array}$ & $\begin{array}{c}\text { Did Exercise } \\
\text { Counteract } \\
\text { Diet-Induced } \\
\text { Memory } \\
\text { Impairment? }\end{array}$ & Mechanisms \\
\hline $\begin{array}{l}\text { Noble et al. } \\
\text { (2014) [32] }\end{array}$ & $\begin{array}{l}\text { 7-month-old Naïve } \\
\text { rats }\end{array}$ & $\begin{array}{l}\text { Forced treadmill or } \\
\text { voluntary wheel } \\
\text { access for } 7 \text { weeks }\end{array}$ & $\begin{array}{c}16 \text { weeks of high-fat } \\
\text { diet }\end{array}$ & $\begin{array}{c}\text { Exercise } \\
\text { occurring after } \\
\text { high-fat diet } \\
\text { (treatment) }\end{array}$ & $\begin{array}{l}\text { Two-way active } \\
\text { avoidance test }\end{array}$ & Yes & Yes & $\begin{array}{c}\text { Increased BDNF in } \\
\text { CA3. }\end{array}$ \\
\hline $\begin{array}{l}\text { Cheng et al. } \\
\text { (2016) [33] }\end{array}$ & $\begin{array}{l}\text { Twelve-week-old } \\
\text { C57BL/6J mice }\end{array}$ & $\begin{array}{c}\text { Treadmill running, } \\
60 \mathrm{~min} / \text { day, } 5 \\
\text { times/week, } 15 \\
\text { m/min, for } 16 \\
\text { weeks. }\end{array}$ & $\begin{array}{c}16 \text { weeks of high-fat } \\
\text { diet ad libitum, } \\
\text { involving } 60 \% \text { fat, } \\
20 \% \text { CHO, and } 20 \% \\
\text { protein }\end{array}$ & Concurrent & $\begin{array}{l}\text { Morris water } \\
\text { maze }\end{array}$ & Yes & Yes & $\begin{array}{l}\text { p-CREB, BACE1, IDE, } \\
\text { and PSD95 were likely } \\
\text { mediators of this effect. }\end{array}$ \\
\hline $\begin{array}{l}\text { Kang et al. } \\
\text { (2016) [34] }\end{array}$ & $\begin{array}{l}\text { Sprague-Dawley } \\
\text { rats aged } 8 \text { weeks }\end{array}$ & $\begin{array}{l}\text { Treadmill running, } \\
30 \mathrm{~min} / \text { day, } 5 \\
\text { days/week, for } 8 \\
\text { weeks. }\end{array}$ & $\begin{array}{l}\text { High fat diet ( } 60 \% \text { fat }) \\
\text { for } 20 \text { weeks }\end{array}$ & $\begin{array}{c}\text { Exercise } \\
\text { occurring after } \\
\text { high-fat diet } \\
\text { (treatment) }\end{array}$ & $\begin{array}{c}\text { Passive } \\
\text { avoidance task }\end{array}$ & Yes & Yes & $\begin{array}{l}\text { Reduction in } \\
\text { pro-inflammatory } \\
\text { cytokines (TNF- } \alpha \text {, } \\
\text { interleukin-1 } \beta \text { ). }\end{array}$ \\
\hline $\begin{array}{c}\text { Kim et al. (2016) } \\
\text { [35] }\end{array}$ & $\begin{array}{c}\text { Male C57BL/6 mice, } \\
\text { 4-weeks old }\end{array}$ & $\begin{array}{l}\text { Treadmill exercise, } \\
\text { ranging from } 30 \text { to } \\
50 \mathrm{~min} / \text { day; } \\
\text { progressive over a } \\
\text { 20-week period. }\end{array}$ & $\begin{array}{l}\text { High-fat diet ( } 60 \% \text { fat) } \\
\text { for } 20 \text { weeks ad } \\
\text { libitum }\end{array}$ & $\begin{array}{c}\text { Exercise } \\
\text { occurring after } \\
\text { high-fat diet } \\
\text { (treatment) }\end{array}$ & $\begin{array}{l}\text { Y-maze test and } \\
\text { radial-8-arm } \\
\text { maze test }\end{array}$ & Yes & Yes & $\begin{array}{l}\text { Increased expression of } \\
\text { BDNF and TrkB and } \\
\text { enhanced cell } \\
\text { proliferation. }\end{array}$ \\
\hline $\begin{array}{l}\text { Klein et al. } \\
\text { (2016) [28] }\end{array}$ & $\begin{array}{l}\text { Six-week-old female } \\
\text { C57Bl/6N mice }\end{array}$ & $\begin{array}{l}\text { Free access to } \\
\text { running wheel. }\end{array}$ & $\begin{array}{l}12 \text { weeks of high-fat } \\
\text { diet, involving } 60 \% \\
\text { fat, } 20 \% \mathrm{CHO} \text {, and } \\
20 \% \text { protein }\end{array}$ & Concurrent & $\begin{array}{l}\text { Morris water } \\
\text { maze }\end{array}$ & $\begin{array}{l}\text { Yes, but only in } \\
\text { adolescent }\end{array}$ & Yes & $\begin{array}{l}\text { Modulation of } \\
\text { hippocampal } \\
\text { neurogenesis. }\end{array}$ \\
\hline $\begin{array}{c}\text { Park et al. (2017) } \\
\text { [36] }\end{array}$ & $\begin{array}{l}\text { Male 4-week-old } \\
\text { C57BL/6 mice }\end{array}$ & $\begin{array}{c}\text { Treadmill exercise, } 6 \\
\text { days/week, approx. } \\
40 \text { min/day, for } 12 \\
\text { weeks. }\end{array}$ & $\begin{array}{l}20 \text { weeks of high-fat } \\
\text { diet, involving } 60 \% \\
\text { fat ad libitum }\end{array}$ & $\begin{array}{c}\text { Exercise } \\
\text { occurring after } \\
\text { high-fat diet } \\
\text { (treatment) }\end{array}$ & $\begin{array}{l}\text { Step-down } \\
\text { avoidance task }\end{array}$ & Yes & Yes & $\begin{array}{l}\text { Reduced insulin } \\
\text { resistance, improved } \\
\text { mitochondrial function, } \\
\text { and reduced apoptosis } \\
\text { in the hippocampus. }\end{array}$ \\
\hline $\begin{array}{l}\text { Cheng et al. } \\
\text { (2018) [37] }\end{array}$ & $\begin{array}{l}\text { Male 3-week-old } \\
\text { SHR and } \\
\text { normotensive } \\
\text { Wistar-Kyoto rats }\end{array}$ & $\begin{array}{l}\text { Swimming exercise } \\
\text { for } 6 \text { weeks. }\end{array}$ & $\begin{array}{c}6 \text { weeks of } \\
\text { low-soybean oil diet }\end{array}$ & Concurrently & $\begin{array}{l}\text { Morris water } \\
\text { maze }\end{array}$ & Yes & Yes & $\begin{array}{l}\text { Up-regulation of BDNF } \\
\text { and NMDA-r } \\
\text { expression. }\end{array}$ \\
\hline
\end{tabular}


Table 1. Cont

\begin{tabular}{|c|c|c|c|c|c|c|c|c|}
\hline Study & Subjects & Exercise Protocol & Diet Protocol & $\begin{array}{c}\text { Temporal } \\
\text { Assessment of } \\
\text { Exercise and } \\
\text { Diet }\end{array}$ & $\begin{array}{l}\text { Memory } \\
\text { Assessment }\end{array}$ & $\begin{array}{l}\text { Did High-Fat } \\
\text { Diet Impair } \\
\text { Memory? }\end{array}$ & $\begin{array}{c}\text { Did Exercise } \\
\text { Counteract } \\
\text { Diet-Induced } \\
\text { Memory } \\
\text { Impairment? }\end{array}$ & Mechanisms \\
\hline $\begin{array}{l}\text { Jeong et al. } \\
\text { (2018) [38] }\end{array}$ & $\begin{array}{l}\text { Sprague-Dawley } \\
\text { rats aged } 20 \text { weeks }\end{array}$ & $\begin{array}{l}\text { Treadmill exercise } \\
\text { for } 8 \text { weeks, } 30 \\
\min / \text { day, } 8 \mathrm{~m} / \mathrm{min}, 5 \\
\text { days/week. }\end{array}$ & $\begin{array}{l}\text { High-fat diet for } 20 \\
\text { weeks }\end{array}$ & $\begin{array}{c}\text { Exercise } \\
\text { occurring after } \\
\text { high-fat diet } \\
\text { (treatment) }\end{array}$ & $\begin{array}{l}\text { Water maze and } \\
\text { passive } \\
\text { avoidance tasks }\end{array}$ & Yes & Yes & $\begin{array}{l}\text { Improved brain insulin } \\
\text { signaling, inhibition of } \\
\text { obesity-induced } \\
\text { NADPH-oxidase } \\
\text { activity. }\end{array}$ \\
\hline $\begin{array}{l}\text { Jeong et al. } \\
\text { (2018) [39] }\end{array}$ & $\begin{array}{l}\text { Sprague-Dawley } \\
\text { rats aged } 8 \text { weeks }\end{array}$ & $\begin{array}{c}\text { Treadmill exercise } \\
\text { for } 8 \text { weeks, } 30 \\
\text { min/day, } 5 \\
\text { days/week, } \\
\text { progressive } \\
\text { intensity. }\end{array}$ & $\begin{array}{l}\text { High fat diet for } 20 \\
\text { weeks, including } 20 \% \\
\text { CHO, } 60 \% \text { fat, and } \\
20 \% \text { protein }\end{array}$ & $\begin{array}{c}\text { Exercise } \\
\text { occurring after } \\
\text { high-fat diet } \\
\text { (treatment) }\end{array}$ & $\begin{array}{c}\text { Passive } \\
\text { avoidance task }\end{array}$ & Yes & Yes & $\begin{array}{c}\text { Improved brain insulin } \\
\text { signaling } \\
\text { (PI3K/AKT/GSK-3ß), } \\
\text { reduced tau } \\
\text { hyperphosphorylation. }\end{array}$ \\
\hline $\begin{array}{c}\text { Shi et al. (2018) } \\
\text { [40] }\end{array}$ & $\begin{array}{l}\text { Male C57BL/6 mice } \\
\text { and SIRT3 mice } \\
\text { (2-months old) }\end{array}$ & $\begin{array}{l}\text { Exercise started at } \\
\text { week } 6 \text { and } \\
\text { continued for the } \\
\text { remaining } 6 \text { weeks. } \\
\text { Engaged in aerobic } \\
\text { intermittent } \\
\text { training, } 30 \mathrm{~min} / \text { day, } \\
5 \text { days/week. } \\
\text { Intermittent } \\
\text { exercise involved } \\
4 \text {-min bursts at } \\
80-85 \% \text { of VO2max, } \\
\text { with } 2 \text { min active } \\
\text { recovery periods. }\end{array}$ & $\begin{array}{l}\text { High-fat diet of } 45 \% \\
\text { kcal fat, } 20 \% \mathrm{kcal} \\
\text { protein, and } 35 \% \mathrm{kcal} \\
\text { CHO for } 12 \text { weeks }\end{array}$ & Concurrent & $\begin{array}{l}\text { Morris water } \\
\text { maze }\end{array}$ & Yes & Yes & $\begin{array}{l}\text { SIRT3 upregulation and } \\
\text { improvement in } \\
\text { antioxidative activity }\end{array}$ \\
\hline $\begin{array}{c}\text { Han et al. (2019) } \\
\text { [41] }\end{array}$ & $\begin{array}{l}\text { Six-week-old } \\
\text { C57BL/6 mice }\end{array}$ & $\begin{array}{c}23 \text { weeks of } \\
\text { treadmill running, } \\
30 \mathrm{~min} / \text { day, } 5 \\
\text { days } / \text { week, at } 8 \\
\mathrm{~m} / \mathrm{min} .\end{array}$ & $\begin{array}{l}23 \text { weeks of high-fat } \\
\text { diet ad libitum, } \\
\text { involving } 60 \% \text { fat }\end{array}$ & Concurrent & $\begin{array}{l}\text { Morris water } \\
\text { maze }\end{array}$ & Yes & Yes & $\begin{array}{l}\text { Reduced number of } \\
\text { apoptotic cells and } \\
\text { increased BDNF. }\end{array}$ \\
\hline $\begin{array}{l}\text { Mehta et al. } \\
(2019) \text { [42] }\end{array}$ & $\begin{array}{l}\text { Sprague-Dawley } \\
\text { male rats }\end{array}$ & $\begin{array}{l}\text { Running wheel } \\
\text { access for } 6 \text { weeks, } \\
\text { 25-30 min/day, } 5 \\
\text { days/week. }\end{array}$ & $\begin{array}{l}15 \text { days of high-fat } \\
\operatorname{diet}(310 \mathrm{gm} / \mathrm{kg} \text { Lard })\end{array}$ & $\begin{array}{c}\text { Exercise } \\
\text { occurring after } \\
\text { high-fat diet } \\
\text { (treatment) }\end{array}$ & $\begin{array}{c}\text { Passive } \\
\text { avoidance and } \\
\text { elevated plus } \\
\text { maze }\end{array}$ & Yes & Yes & $\begin{array}{c}\text { Reduction in } \\
\text { neuroinflammatory } \\
\text { markers (e.g., IL-1 } \beta \text {, } \\
\text { TNF- } \alpha \text { ). }\end{array}$ \\
\hline
\end{tabular}




\section{Discussion}

The present review examines whether exercise can counteract HFD-induced memory impairment. Main findings from the present review are twofold: (1) chronic HFD robustly impairs memory function, and (2) chronic exercise engagement, occurring either concurrently or after the diet protocol, robustly counteracted HFD-induced memory impairment. This latter finding occurred among studies that employed various exercise protocols, such as voluntary access to a running wheel or forced exercise on a treadmill. Similarly, across these studies, the exercise protocol varied from 6 to 23 weeks. Further, various spatial-related memory tasks were employed across the evaluated studies. Despite these variations in the exercise protocols and memory tasks, exercise robustly counteracted HFD-induced memory impairment.

A mechanism through which exercise may counteract HFD-induce memory impairment is likely through alterations in processes related to synaptic transmission and production of plasticity-related proteins. As thoroughly addressed elsewhere [43-48], LTP involves several phases, including early-LTP (E-LTP) and late-LTP (L-LTP) [47]. In brief, E-LTP, a protein synthesis-independent process, involves the activation of several kinases (e.g., PKA, CaMKII), which play a critical role in phosphorylating proteins and receptors (e.g., AMPA, NDMA), eventually potentiating synaptic transmission [47]. Endocytosis of such receptors, via, for example, phosphatase activity, may induce LTD [10]. In contrast to E-LTP, L-LTP, a protein synthesis-dependent process, involves gene expression and local protein synthesis via, for example, the TrkB receptor [47]. The following paragraphs link some of these processes to episodic memory function, how HFD impairs these processes, and how exercise influences these processes.

As noted in Table 1 and as shown in Figure 1, potential mechanisms of this exercise-related counteraction effect of HFD-induced memory impairment are multifold. Such effects may include exercise-induced alterations in some of the above-mentioned pathways. For example, activation of the BDNF receptor, TrkB, plays an important role in spatial memory [49]. Specifically, BDNF appears to play a critical role in the consolidation of memories, as previous work demonstrates that continuous intracerebroventricular infusion of antisense BDNF oligonucleotide causes spatial memory deficit [50]. An HFD has been shown to reduce hippocampal BDNF levels and downstream effectors [20], which may lower the neurochemical substrate of the hippocampus that is needed for optimal neuronal function. Exercise may counteract this HFD-induced BDNF reduction and memory impairment via its role in augmenting BDNF levels, via $\beta$-hydroxybutyrate alteration [51]. Exercise-induced increases in $\beta$-hydroxybutyrate are thought to inhibit histone deacetylases, ultimately facilitating hippocampal BDNF expression [51].

In addition to BDNF, synapsin 1, a neuronal phosphoprotein, plays an important role in regulating neurotransmitter release. A chronic HFD has been shown to lower synapsin 1 levels [20] and reduction of synapsin 1 leads to spatial memory deficit [52,53]. Exercise has been shown to increase synapsin 1 levels [54], which is likely occurring from exercise-induced increases in BDNF (i.e., BDNF may promote the phosphorylation of synapsin 1) [55]. BDNF also plays an important role in hippocampal neurogenesis [56], which may play a causal role in spatial memory. Ablation of adult hippocampal neurogenesis results in impairment of acquiring spatial reference memory [57]. Neurogenesis plays an important role in spatial memory and may, for example, occur via pattern separation mechanisms [58]. A chronic HFD may impair neurogenesis through increases in corticosterone [59], with exercise potentially counteracting this effect via BDNF-mediated hippocampal neurogenesis [60].

In conclusion, this review demonstrated that episodic memory may be impaired with a chronic HFD, yet this effect may be counteracted by chronic engagement in exercise. Future work should consider this model in the context of a preventive paradigm. All of the evaluated studies in this review employed a concurrent or treatment-based model and, thus, it would be worthwhile to evaluate if a period of exercise prior to an HFD protocol can counteract the detrimental effects of an HFD on memory function. Furthermore, future work should also consider evaluating other memory systems (e.g., working memory, episodic memory, procedural memory, prospective memory) to determine whether the observed associations hold true across different memory systems. 


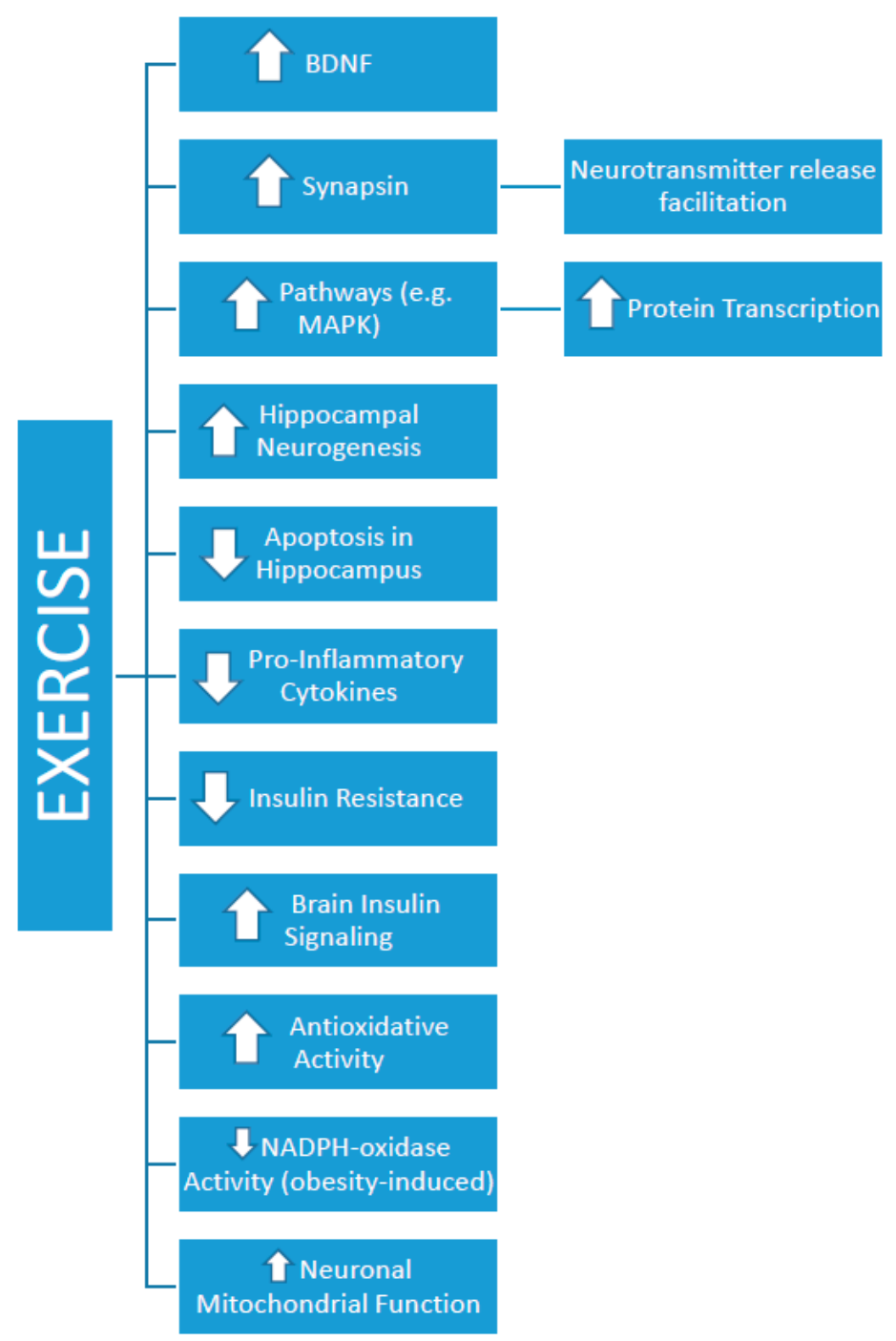

Figure 1. Schematic illustrating the mechanistic role through which exercise may counteract a high-fat diet-induced impairment of memory function.

Funding: This research project is supported by both Guangdong-Government Funding for Scientific Research (2016KZDXM009) and Shenzhen-Government Research Grants Programme in Basic Sciences (JCYJ20150729104249783).

Conflicts of Interest: The authors declare no conflict of interest.

\section{References}

1. DeSalvo, K.B.; Olson, R.; Casavale, K.O. Dietary Guidelines for Americans. JAMA 2016, 315, 1. [CrossRef] [PubMed]

2. Webster, C.C.; Swart, J.; Noakes, T.D.; Smith, J.A. A Carbohydrate Ingestion Intervention in an Elite Athlete Who Follows a Low-Carbohydrate High-Fat Diet. Int. J. Sports Physiol. Perform. 2018, 13, 957-960. [CrossRef] [PubMed]

3. Noakes, T.D.; Windt, J. Evidence that supports the prescription of low-carbohydrate high-fat diets: A narrative review. Br. J. Sports Med. 2017, 51, 133-139. [CrossRef]

4. Molteni, R.; Wu, A.; Vaynman, S.; Ying, Z.; Barnard, R.; Gómez-Pinilla, F. Exercise reverses the harmful effects of consumption of a high-fat diet on synaptic and behavioral plasticity associated to the action of brain-derived neurotrophic factor. Neuroscience 2004, 123, 429-440. [CrossRef] 
5. Maesako, M.; Uemura, K.; Kubota, M.; Kuzuya, A.; Sasaki, K.; Asada, M.; Watanabe, K.; Hayashida, N.; Ihara, M.; Ito, H.; et al. Environmental enrichment ameliorated high-fat diet-induced A $\beta$ deposition and memory deficit in APP transgenic mice. Neurobiol. Aging 2012, 33, 1011.e11-1011.e23. [CrossRef]

6. Tulving, E. Elements of Episodic Memory; Oxford University Press: New York, NY, USA, 1983.

7. Mishkin, M.; Suzuki, W.A.; Gadian, D.G.; Vargha-Khadem, F.; Price, T. Hierarchical organization of cognitive memory. Philos. Trans. R. Soc. B Boil. Sci. 1997, 352, 1461-1467. [CrossRef]

8. Poo, M.-M.; Pignatelli, M.; Ryan, T.J.; Tonegawa, S.; Bonhoeffer, T.; Martin, K.C.; Rudenko, A.; Tsai, L.-H.; Tsien, R.W.; Fishell, G.; et al. What is memory? The present state of the engram. BMC Boil. 2016, 14, 1133. [CrossRef] [PubMed]

9. Bliss, T.V.P.; Lømo, T. Long-lasting potentiation of synaptic transmission in the dentate area of the anaesthetized rabbit following stimulation of the perforant path. J. Physiol. 1973, 232, 331-356. [CrossRef]

10. Bol'shakov, V. Mechanisms of long-term synaptic depression in the hippocampus. Rossiiskii Fiziologicheskii Zhurnal Imeni I.M. Sechenova 2001, 87, 441-447.

11. Doyère, V.; Debiec, J.; Monfils, M.-H.; E Schafe, G.; E LeDoux, J. Synapse-specific reconsolidation of distinct fear memories in the lateral amygdala. Nat. Neurosci. 2007, 10, 414-416. [CrossRef]

12. Whitlock, J.R.; Heynen, A.J.; Shuler, M.G.; Bear, M.F. Learning induces long-term potentiation in the hippocampus. Science 2006, 313, 1093-1097. [CrossRef]

13. Gruart, A.; Muñoz, M.D.; Delgado-García, J.M. Involvement of the CA3-CA1 Synapse in the Acquisition of Associative Learning in Behaving Mice. J. Neurosci. 2006, 26, 1077-1087. [CrossRef] [PubMed]

14. Hao, S.; Dey, A.; Yu, X.; Stranahan, A.M. Dietary obesity reversibly induces synaptic stripping by microglia and impairs hippocampal plasticity. Brain Behav. Immun. 2016, 51, 230-239. [CrossRef] [PubMed]

15. Valladolid-Acebes, I.; Merino, B.; Principato, A.; Fole, A.; Barbas, C.; Lorenzo, M.P.; Del Olmo, N.; Ruiz-Gayo, M.; Cano, V.; Garcia, A. High-fat diets induce changes in hippocampal glutamate metabolism and neurotransmission. Am. J. Physiol. Metab. 2012, 302, 396-402. [CrossRef] [PubMed]

16. Karimi, S.A.; Salehi, I.; Komaki, A.; Sarihi, A.; Zarei, M.; Shahidi, S. Effect of high-fat diet and antioxidants on hippocampal long-term potentiation in rats: An in vivo study. Brain Res. 2013, 1539, 1-6. [CrossRef] [PubMed]

17. Wang, Z.; Fan, J.; Wang, J.; Li, Y.; Xiao, L.; Duan, D.; Wang, Q. Protective effect of lycopene on high-fat diet-induced cognitive impairment in rats. Neurosci. Lett. 2016, 627, 185-191. [CrossRef]

18. Cano, V.; Valladolid-Acebes, I.; Hernández-Nuño, F.; Merino, B.; Del Olmo, N.; Chowen, J.A.; Ruiz-Gayo, M. Morphological changes in glial fibrillary acidic protein immunopositive astrocytes in the hippocampus of dietary-induced obese mice. NeuroReport 2014, 25, 819-822. [CrossRef] [PubMed]

19. Page, K.C.; Jones, E.K.; Anday, E.K. Maternal and postweaning high-fat diets disturb hippocampal gene expression, learning, and memory function. Am. J. Physiol. Integr. Comp. Physiol. 2014, 306, 527-537. [CrossRef] [PubMed]

20. Molteni, R.; Barnard, R.; Ying, Z.; Roberts, C.; Gómez-Pinilla, F. A high-fat, refined sugar diet reduces hippocampal brain-derived neurotrophic factor, neuronal plasticity, and learning. Neuroscience 2002, 112, 803-814. [CrossRef]

21. Tozuka, Y.; Kumon, M.; Wada, E.; Onodera, M.; Mochizuki, H.; Wada, K. Maternal obesity impairs hippocampal BDNF production and spatial learning performance in young mouse offspring. Neurochem. Int. 2010, 57, 235-247. [CrossRef]

22. Cassilhas, R.; Lee, K.; Fernandes, J.; Oliveira, M.; Tufik, S.; Meeusen, R.; De Mello, M. Spatial memory is improved by aerobic and resistance exercise through divergent molecular mechanisms. Neuroscience 2012, 202, 309-317. [CrossRef] [PubMed]

23. Van Praag, H.; Christie, B.R.; Sejnowski, T.J.; Gage, F.H. Running enhances neurogenesis, learning, and long-term potentiation in mice. Proc. Natl. Acad. Sci. USA 1999, 96, 13427-13431. [CrossRef] [PubMed]

24. Huang, Y.-Q.; Wu, C.; He, X.-F.; Wu, D.; He, X.; Liang, F.-Y.; Dai, G.-Y.; Pei, Z.; Xu, G.-Q.; Lan, Y. Effects of Voluntary Wheel-Running Types on Hippocampal Neurogenesis and Spatial Cognition in Middle-Aged Mice. Front. Cell. Neurosci. 2018, 12, 177. [CrossRef] [PubMed]

25. Molteni, R.; Ying, Z.; Gomez-Pinilla, F.; Gómez-Pinilla, F. Differential effects of acute and chronic exercise on plasticity-related genes in the rat hippocampus revealed by microarray. Eur. J. Neurosci. 2002, 16, 1107-1116. [CrossRef] [PubMed] 
26. Dietrich, M.O.; Mantese, C.E.; Porciúncula, L.O.; Ghisleni, G.; Vinade, L.; Souza, D.O.; Portela, L.V. Exercise affects glutamate receptors in postsynaptic densities from cortical mice brain. Brain Res. 2005, 1065, $20-25$. [CrossRef] [PubMed]

27. Bramer, W.M.; Rethlefsen, M.L.; Kleijnen, J.; Franco, O.H. Optimal database combinations for literature searches in systematic reviews: A prospective exploratory study. Syst. Rev. 2017, 6, 245. [CrossRef] [PubMed]

28. Klein, C.; Jonas, W.; Iggena, D.; Empl, L.; Rivalan, M.; Wiedmer, P.; Spranger, J.; Hellweg, R.; Winter, Y.; Steiner, B. Exercise prevents high-fat diet-induced impairment of flexible memory expression in the water maze and modulates adult hippocampal neurogenesis in mice. Neurobiol. Learn. Mem. 2016, 131, $26-35$. [CrossRef]

29. Maesako, M.; Uemura, K.; Iwata, A.; Kubota, M.; Watanabe, K.; Uemura, M.; Noda, Y.; Asada-Utsugi, M.; Kihara, T.; Takahashi, R.; et al. Continuation of Exercise Is Necessary to Inhibit High Fat Diet-Induced $\beta$-Amyloid Deposition and Memory Deficit in Amyloid Precursor Protein Transgenic Mice. PLoS ONE 2013, 8, e72796. [CrossRef]

30. Maesako, M.; Uemura, K.; Kubota, M.; Kuzuya, A.; Sasaki, K.; Hayashida, N.; Asada-Utsugi, M.; Watanabe, K.; Uemura, M.; Kihara, T.; et al. Exercise Is More Effective than Diet Control in Preventing High Fat Diet-induced $\beta$-Amyloid Deposition and Memory Deficit in Amyloid Precursor Protein Transgenic Mice. J. Boil. Chem. 2012, 287, 23024-23033. [CrossRef]

31. Woo, J.; Shin, K.O.; Park, S.Y.; Jang, K.S.; Kang, S. Effects of exercise and diet change on cognition function and synaptic plasticity in high fat diet induced obese rats. Lipids Heal. Dis. 2013, 12, 144. [CrossRef]

32. Noble, E.E.; Mavanji, V.; Little, M.R.; Billington, C.J.; Kotz, C.M.; Wang, C. Exercise reduces diet-induced cognitive decline and increases hippocampal brain-derived neurotrophic factor in CA3 neurons. Neurobiol. Learn. Mem. 2014, 114, 40-50. [CrossRef] [PubMed]

33. Cheng, J.; Chen, L.; Han, S.; Qin, L.; Chen, N.; Wan, Z. Treadmill Running and Rutin Reverse High Fat Diet Induced Cognitive Impairment in Diet Induced Obese Mice. J. Nutr. Health Aging 2016, 20, 503-508. [CrossRef] [PubMed]

34. Kang, E.; Koo, J.; Jang, Y.; Yang, C.; Lee, Y.; Cosio-Lima, L.M.; Cho, J. Neuroprotective Effects of Endurance Exercise against High Fat Diet-Induced Hippocampal Neuroinflammation. J. Neuroendocr. 2016, 28, 28. [CrossRef] [PubMed]

35. Kim, T.-W.; Choi, H.-H.; Chung, Y.-R. Treadmill exercise alleviates impairment of cognitive function by enhancing hippocampal neuroplasticity in the high-fat diet-induced obese mice. J. Exerc. Rehabil. 2016, 12, 156-162. [CrossRef] [PubMed]

36. Park, H.S.; Cho, H.S.; Kim, T.W. Physical exercise promotes memory capability by enhancing hippocampal mitochondrial functions and inhibiting apoptosis in obesity-induced insulin resistance by high fat diet. Metab. Brain Dis. 2018, 33, 283-292. [CrossRef] [PubMed]

37. Cheng, M.; Cong, J.; Wu, Y.; Xie, J.; Wang, S.; Zhao, Y.; Zang, X. Chronic Swimming Exercise Ameliorates Low-Soybean-Oil Diet-Induced Spatial Memory Impairment by Enhancing BDNF-Mediated Synaptic Potentiation in Developing Spontaneously Hypertensive Rats. Neurochem. Res. 2018, 43, 1047-1057. [CrossRef] [PubMed]

38. Jeong, J.-H.; Koo, J.-H.; Cho, J.-Y.; Kang, E.-B. Neuroprotective effect of treadmill exercise against blunted brain insulin signaling, NADPH oxidase, and Tau hyperphosphorylation in rats fed a high-fat diet. Brain Res. Bull. 2018, 142, 374-383. [CrossRef]

39. Jeong, J.-H.; Kang, E.-B. Effects of treadmill exercise on PI3K/AKT/GSK-3 $\beta$ pathway and tau protein in high-fat diet-fed rats. J. Exerc. Nutr. Biochem. 2018, 22, 9-14. [CrossRef] [PubMed]

40. Shi, Z.; Li, C.; Yin, Y.; Yang, Z.; Xue, H.; Mu, N.; Wang, Y.; Liu, M.; Ma, H. Aerobic Interval Training Regulated SIRT3 Attenuates High-Fat-Diet-Associated Cognitive Dysfunction. BioMed Res. Int. 2018, 2018, 1-8. [CrossRef]

41. Han, T.-K.; Leem, Y.-H.; Kim, H.-S. Treadmill exercise restores high fat diet-induced disturbance of hippocampal neurogenesis through $\beta 2$-adrenergic receptor-dependent induction of thioredoxin-1 and brain-derived neurotrophic factor. Brain Res. 2019, 1707, 154-163. [CrossRef]

42. Mehta, B.K.; Singh, K.K.; Banerjee, S. Effect of exercise on type 2 diabetes-associated cognitive impairment in rats. Int. J. Neurosci. 2019, 129, 252-263. [CrossRef] [PubMed]

43. Blitzer, R.D. Teaching resources. Long-term potentiation: Mechanisms of induction and maintenance. Sci. STKE 2005, 2005, tr26. [CrossRef] [PubMed] 
44. Sweatt, J.D. Toward a Molecular Explanation for Long-Term Potentiation. Learn. Mem. 1999, 6, $399-416$. [CrossRef] [PubMed]

45. Buonarati, O.R.; Hammes, E.A.; Watson, J.F.; Greger, I.H.; Hell, J.W. Mechanisms of postsynaptic localization of AMPA-type glutamate receptors and their regulation during long-term potentiation. Sci. Signal. 2019, 12, eaar6889. [CrossRef] [PubMed]

46. Sacktor, T.C.; Fenton, A.A. What does LTP tell us about the roles of CaMKII and PKMzeta in memory? Mol. Brain 2018, 11, 77. [CrossRef] [PubMed]

47. Baltaci, S.B.; Mogulkoc, R.; Baltacim, A.K. Molecular Mechanisms of Early and Late LTP. Neurochem. Res. 2019, 44, 281-296. [CrossRef] [PubMed]

48. Park, M. AMPA Receptor Trafficking for Postsynaptic Potentiation. Front. Cell. Neurosci. 2018, $12,361$. [CrossRef] [PubMed]

49. Mizuno, M.; Yamada, K.; He, J.; Nakajima, A.; Nabeshima, T. Involvement of BDNF Receptor TrkB in Spatial Memory Formation. Learn. Mem. 2003, 10, 108-115. [CrossRef] [PubMed]

50. Mizuno, M.; Yamada, K.; Olariu, A.; Nawa, H.; Nabeshima, T. Involvement of Brain-Derived Neurotrophic Factor in Spatial Memory Formation and Maintenance in a Radial Arm Maze Test in Rats. J. Neurosci. 2000, 20, 7116-7121. [CrossRef] [PubMed]

51. Sleiman, S.F.; Henry, J.; Al-Haddad, R.; El Hayek, L.; Haidar, E.A.; Stringer, T.; Ulja, D.; Karuppagounder, S.S.; Holson, E.B.; Ratan, R.R.; et al. Exercise promotes the expression of brain derived neurotrophic factor (BDNF) through the action of the ketone body $\beta$-hydroxybutyrate. eLife 2016, 5, 5. [CrossRef] [PubMed]

52. Qiao, S.; Peng, R.; Yan, H.; Gao, Y.; Wang, C.; Wang, S.; Zou, Y.; Xu, X.; Zhao, L.; Dong, J.; et al. Reduction of Phosphorylated Synapsin I (Ser-553) Leads to Spatial Memory Impairment by Attenuating GABA Release after Microwave Exposure in Wistar Rats. PLoS ONE 2014, 9, e95503. [CrossRef] [PubMed]

53. John, J.P.P.; Sunyer, B.; Höger, H.; Pollak, A.; Lubec, G. Hippocampal synapsin isoform levels are linked to spatial memory enhancement by SGS742. Hippocampus 2009, 19, 731-738. [CrossRef] [PubMed]

54. Vaynman, S.; Ying, Z.; Gómez-Pinilla, F.; Gómez-Pinilla, F. Exercise induces BDNF and synapsin I to specific hippocampal subfields. J. Neurosci. Res. 2004, 76, 356-362. [CrossRef]

55. Jovanovic, J.N.; Czernik, A.J.; Fienberg, A.A.; Greengard, P.; Sihra, T.S. Synapsins as mediators of BDNF-enhanced neurotransmitter release. Nat. Neurosci. 2000, 3, 323-329. [CrossRef] [PubMed]

56. Rossi, C.; Angelucci, A.; Costantin, L.; Braschi, C.; Mazzantini, M.; Babbini, F.; Fabbri, M.E.; Tessarollo, L.; Maffei, L.; Berardi, N.; et al. Brain-derived neurotrophic factor (BDNF) is required for the enhancement of hippocampal neurogenesis following environmental enrichment. Eur. J. Neurosci. 2006, 24, 1850-1856. [CrossRef] [PubMed]

57. Dupret, D.; Revest, J.-M.; Koehl, M.; Ichas, F.; De Giorgi, F.; Costet, P.; Abrous, D.N.; Piazza, P.V. Spatial Relational Memory Requires Hippocampal Adult Neurogenesis. PLoS ONE 2008, 3, e1959. [CrossRef]

58. França, T.F.A.; Bitencourt, A.M.; Maximilla, N.R.; Barros, D.M.; Monserrat, J.M. Hippocampal neurogenesis and pattern separation: A meta-analysis of behavioral data. Hippocampus 2017, 27, 937-950. [CrossRef]

59. Lindqvist, A.; Mohapel, P.; Bouter, B.; Frielingsdorf, H.; Pizzo, D.; Brundin, P.; Erlanson-Albertsson, C.; Erlanson-Albertsson, C.; Erlanson-Albertsson, C. High-fat diet impairs hippocampal neurogenesis in male rats. Eur. J. Neurol. 2006, 13, 1385-1388. [CrossRef]

60. Lee, J.; Duan, W.; Mattson, M.P. Evidence that brain-derived neurotrophic factor is required for basal neurogenesis and mediates, in part, the enhancement of neurogenesis by dietary restriction in the hippocampus of adult mice. J. Neurochem. 2002, 82, 1367-1375. [CrossRef]

(C) 2019 by the authors. Licensee MDPI, Basel, Switzerland. This article is an open access article distributed under the terms and conditions of the Creative Commons Attribution (CC BY) license (http://creativecommons.org/licenses/by/4.0/). 